\title{
Response to Sodium Channel blocking Antiseizure medications and coding polymorphisms of Sodium Channel genes in Taiwanese epilepsy patients
}

Chih-Hsiang Lin ${ }^{1}$, Chen-Jui Ho ${ }^{1}$, Yan-Ting Lu' ${ }^{1}$ and Meng-Han Tsai ${ }^{1,2^{*}}$

\begin{abstract}
Background: Many antiseizure medications (ASMs) control seizures by blocking voltage-dependent sodium channels. Polymorphisms of sodium channel genes may affect the response to ASMs due to altering the effect of ASMs on blocking sodium channels.

Methods: We conducted a retrospective study of epilepsy patients followed up at the Neurological Department of Kaohsiung Chang Gung Memorial Hospital, Taiwan between January 2010 and December 2018. We categorized the patients into response, partial response, and failure to sodium channel blocking ASM groups. Sodium channel blocking ASMs included phenytoin, carbamazepine, lamotrigine, oxcarbazepine, lacosamide, zonisamide, topiramate, and valproic acid. A subgroup of predominant sodium channel blocking ASMs included phenytoin, carbamazepine, lamotrigine, oxcarbazepine, and lacosamide. Associations between the response of ASMs and single-nucleotide polymorphisms of SCN1A, SCN1B, SCN2A, and SCN9A were analyzed.

Results: Two hundred Taiwanese patients and 21 single-nucleotide polymorphisms among SCN1A, SCN1B, SCN2A, and SCN9A were evaluated. We found allele C of rs55742440 in SCN1B was statistically significantly associated with not achieving seizure-free with sodium channel blocking ASMs. For the predominant sodium channel blocking ASMs group, no SNPs were associated with the response of ASMs.

Conclusion: Single-nucleotide polymorphism in SCN1B was associated with the response to sodium channel blocking ASMs. This highlights the possibility that beta subunits may affect the function of sodium channels and resulted in different responsiveness to ASMs.
\end{abstract}

Keywords: Antiseizure medications, Sodium channel gene, Single-nucleotide polymorphisms, Drug resistance epilepsy, SCN1B

\footnotetext{
* Correspondence: menghan@cgmh.org.tw

'Department of Neurology, Kaohsiung Chang Gung Memorial Hospital,

Colleague of Medicine, Chang Gung University, Kaohsiung, Kaohsiung City

83301, Taiwan

${ }^{2}$ School of Medicine, College of Medicine, Chang Gung University, Taoyuan,

Taiwan
}

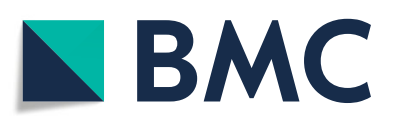

( The Author(s). 2021 Open Access This article is licensed under a Creative Commons Attribution 4.0 International License, which permits use, sharing, adaptation, distribution and reproduction in any medium or format, as long as you give appropriate credit to the original author(s) and the source, provide a link to the Creative Commons licence, and indicate if changes were made. The images or other third party material in this article are included in the article's Creative Commons licence, unless indicated otherwise in a credit line to the material. If material is not included in the article's Creative Commons licence and your intended use is not permitted by statutory regulation or exceeds the permitted use, you will need to obtain permission directly from the copyright holder. To view a copy of this licence, visit http://creativecommons.org/licenses/by/4.0/. The Creative Commons Public Domain Dedication waiver (http://creativecommons.org/publicdomain/zero/1.0/) applies to the data made available in this article, unless otherwise stated in a credit line to the data. 


\section{Background}

Epilepsy is a chronic disorder that requires the longterm use of antiseizure medications (ASMs), the choice of ASM is based on the seizure type and epileptic syndrome, and there are currently no reliable biomarkers to predict the responsiveness to ASMs [1]. With the current advance in the development of ASMs, one-third of patients still have seizures despite multiple ASM treatments [2]. According to the International League Against Epilepsy, drug-resistant epilepsy is defined as the failure of adequate trials of two tolerated, appropriately chosen and used ASM schedules (whether as monotherapies or in combination) to achieve sustained seizure freedom [3]. Several theories tried to explain the cause of drug-resistant epilepsy, including the transporter hypothesis [4], the neuronal network hypothesis [5], the intrinsic severity hypothesis [6], the target hypothesis [7], and the gene variant hypothesis [8].

Among these theories, we focused on the gene variant hypothesis, which is the pharmacogenetic association of the responsiveness of ASM and the genetic variant of ASM targets. Sodium channel (SCN) is responsible for the generation and propagation of action potential in neurons, thus many ASMs act by reducing the highfrequency firing of the voltage-dependent $\mathrm{SCN}$ that occurred during the seizure [9]. SCN is formed by one alpha subunit and two beta subunits. Alpha subunit functions as the voltage sensor and forms the pore region of the channel, while beta subunits regulate or assist the function of SCN [10]. The alpha subunits are encoded by the $S C N(1-10) A$ genes and beta subunits by $S C N(1-4) B$ genes. Many studies had investigated the relationship between $\mathrm{SCN}$ gene single-nucleotide polymorphisms (SNPs) and drug-resistant epilepsy. Tate et al. reported that the SCN1A rs3812718 variant was associated with the maximum dose of phenytoin and carbamazepine for controlling seizures [11]. This SNP has also been reported to be related to drug-resistant epilepsy in different ethnic groups [12-14] but with conflicting results [15-17]. Other SNPs of SCN genes have also been evaluated, but not as extensively [18-20]. A recent study evaluated 39 polymorphisms in the $S C N 1 A$, $S C N 2 A$, and $S C N 3 A$ genes in patients from Malaysia and Hong Kong, had found no associations between gene polymorphisms and responsiveness to ASMs [21].

The above mentioned studies used a selective approach by focusing on genotyping a dozen "common" SNPs, many of which were located in introns or non-coding regions (such as UTR or inter-gene areas), and therefore the interpretation of the functional consequence remains unclear. In this study, we adopted a different approach by using next-generation sequencing-based techniques to cover all coding regions of four sodium channel genes. Using this approach, we could evaluate associations between the responsiveness to ASMs and all coding SNPs, which are more likely to have a functional impact.

\section{Methods \\ Study design}

We retrospectively reviewed the medical records of all patients treated and followed up for epilepsy at the Neurological Department of Kaohsiung Chang Gung Memorial Hospital, Taiwan between January 2010 and December 2018. This study was approved by the Chang Gung Medical Foundation Institutional Review Board (IRB No.: 104-1961C, 104-2308B, 201800433B0D001, and 201901274B0D001).

\section{Definitions and criteria}

The inclusion criteria were patients with epilepsy aged > 20 years who took at least one SCN blocking ASM and followed up for at least 12 months. The SCN blocking ASMs (SCN-ASMs) investigated in this study included phenytoin, carbamazepine, lamotrigine, oxcarbazepine, lacosamide, zonisamide, topiramate, and valproic acid [22]. Since some SCN-ASMs also have broad-spectrum non-SCN mechanisms, such as valproate acid, topiramate, and zonisamide, we further divided the ASMs into a "predominant SCN blocking ASM (predominant SCNASM)" subgroup composed of phenytoin, carbamazepine, lamotrigine, oxcarbazepine, and lacosamide [22]. Patients were excluded if they had a history of psychogenic nonepileptic seizures, those who could not provide information about seizure frequency, those who did not take any SCNASMs, and those with poor drug compliance. Patients with epilepsy syndromes that involved a known genetic mutation of SCN genes or were known to be refractory to multiple ASMs, such as Dravet syndrome and Lennox Gastaut syndrome, were also excluded [23, 24].

We evaluated the efficacy of the ASMs according to the seizure frequency after adding the last SCN-ASM and categorized the efficacy according to the International League Against Epilepsy (ILAE) consensus [3]. A response to the $\mathrm{SCN}$-ASMs was defined as seizure freedom lasting for $\geq 12$ months after taking the last SCN-ASM. Patients not achieving seizure-free were further divided into partial response and failure to SCN-ASMs. A partial response was defined as a seizure frequency $<50 \%$ of the pretreatment seizure frequency during the use of the last SCN-ASM with an adequate trial. Failure to the SCN-ASMs was defined as persistent seizures at $>50 \%$ of the pretreatment seizure frequency after the last SCN-ASM with an adequate trial. If the SCN-ASM trial was stopped before the outcome was known or if the data required to assess the outcomes were missing, the response was categorized as being "unknown" [25]. The classifications for the efficacy of the predominant SCN-ASMs were the same as for the SCN-ASMs. 
Clinical data including gender, onset age, type of seizure, etiology of seizure, seizure frequency, other medical diseases, the type and maintenance dose of ASM, electroencephalography, and brain imaging were collected. Seizure types and epilepsy syndromes were classified according to the 2017 ILAE classification and terminology [26, 27].
Targeted next-generation sequencing gene panels and calling of SNPs

Genomic DNA was extracted from peripheral blood leukocytes using QIAGEN DNA extraction kits (Qiagen, Germany), according to the manufacturer's instructions. A customized panel including four SCN genes (SCN1A,

Table 1 Demographic data of patients taking sodium channel blocking antiseizure medications

\begin{tabular}{|c|c|c|c|c|}
\hline & All $(n=200)$ & Response $(n=109)$ & Not seizure-free $(n=91)$ & $p$ \\
\hline Onset age (year) & $16.0(8.0-24)$ & $17.5(10.0-26.0)$ & $12.8(5.5-20)$ & 0.001 \\
\hline Male & $100(50.0)$ & $54(49.5)$ & $46(50.5)$ & reference \\
\hline Female & $100(50.0)$ & $55(50.5)$ & $45(49.5)$ & 0.887 \\
\hline \multicolumn{5}{|l|}{ Seizure type } \\
\hline Focal & $121(60.5)$ & $62(56.9)$ & $59(64.8)$ & \multirow[t]{3}{*}{0.265} \\
\hline Generalized & $51(25.5)$ & $24(22.0)$ & $27(29.7)$ & \\
\hline Unspecified & $28(14.0)$ & $23(21.1)$ & $5(5.5)$ & \\
\hline \multicolumn{5}{|l|}{ Etiology } \\
\hline Structural & $38(19.0)$ & $17(15.6)$ & $21(23.1)$ & \multirow[t]{5}{*}{0.760} \\
\hline CNS infection & $12(6.0)$ & $6(5.5)$ & $6(6.6)$ & \\
\hline Genetic & $4(2.0)$ & $1(0.9)$ & $3(3.3)$ & \\
\hline Autoimmune & $4(2.0)$ & $1(0.9)$ & $3(3.3)$ & \\
\hline Unknown & $142(71.0)$ & $84(77.1)$ & $58(63.7)$ & \\
\hline \multicolumn{5}{|l|}{ Number of ASMs } \\
\hline 1 & $65(32.5)$ & $58(53.2)$ & $7(7.7)$ & \\
\hline 2 & $61(30.5)$ & $37(33.9)$ & $24(26.4)$ & \\
\hline 3 & $47(23.5)$ & $12(11.0)$ & 35 (38.5) & \\
\hline 4 & $16(8.0)$ & $2(1.8)$ & $14(15.4)$ & \\
\hline 5 & $9(4.5)$ & $0(0.0)$ & $9(9.9)$ & \\
\hline 6 & $2(1.0)$ & $0(0.0)$ & $2(2.2)$ & \\
\hline \multicolumn{5}{|l|}{ Concurrent ASMs } \\
\hline Carbamazepine & 49 & 26 & 23 & \\
\hline Clobazam & 11 & 2 & 9 & \\
\hline Gabapentin & 2 & 0 & 2 & \\
\hline Lacosamide & 3 & 0 & 3 & \\
\hline Lamotrigine & 70 & 35 & 35 & \\
\hline Levetiracetam & 80 & 26 & 54 & \\
\hline Oxcarbazepine & 22 & 7 & 15 & \\
\hline Perampanel & 11 & 0 & 11 & \\
\hline Phenobarbital & 19 & 1 & 12 & \\
\hline Phenytoin & 40 & 22 & 18 & \\
\hline Pregabalin & 3 & 0 & 3 & \\
\hline Topiramate & 41 & 13 & 28 & \\
\hline Valproic acid & 72 & 40 & 32 & \\
\hline Vigabatrin & 4 & 0 & 4 & \\
\hline Zonisamide & 28 & 4 & 24 & \\
\hline
\end{tabular}

Continuous variables were presented as median (interquartile range) Categorical variables were presented as $\mathrm{n}(\%)$ 
$S C N 1 B, S C N 2 A, S C N 9 A)$ was used to sequence all of the coding regions including at least 10 base pairs (bp) flanking sequences of the intron/exon boundaries (primers available upon request). The library was prepared using a multiplex polymerase chain reaction to amplify target regions and then sequenced using an
Illumina MiSeq platform with $2 \times 300 \mathrm{bp}$ paired-end runs. Raw read data were processed with standard bioinformatics pipelines using a Galaxy platform. Briefly, the reads were mapped to the human reference genome (GRCh37) with BWA-MEM and called using FreeBayes. Variants were annotated with wANNOVAR. Only

Table 2 Demographic data of the patients with partial response and failure to sodium channel blocking antiseizure medications

\begin{tabular}{|c|c|c|c|c|}
\hline & All $(n=91)$ & Partial response $(n=23)$ & Failure $(n=68)$ & $p$ \\
\hline Onset age (year) & $12.8(4.5-20.0)$ & $11.0(3.0-19.5)$ & $13(6.0-20.0)$ & 0.496 \\
\hline Male & $46(50.5)$ & $11(47.8)$ & $35(51.5)$ & reference \\
\hline Female & $45(49.5)$ & $12(52.2)$ & $33(48.5)$ & 0.476 \\
\hline \multicolumn{5}{|l|}{ Seizure type } \\
\hline Focal & $59(64.8)$ & $14(60.9)$ & $45(66.2)$ & \multirow[t]{3}{*}{0.811} \\
\hline Generalized & $27(29.7)$ & $8(34.8)$ & 19 (27.9) & \\
\hline Unspecified & $5(5.5)$ & $1(4.3)$ & $4(5.9)$ & \\
\hline \multicolumn{5}{|l|}{ Etiology } \\
\hline Structural & $21(23.1)$ & $5(21.7)$ & $16(23.5)$ & \multirow[t]{5}{*}{0.659} \\
\hline CNS infection & $6(6.6)$ & $2(8.7)$ & $4(5.9)$ & \\
\hline Genetic & $3(3.3)$ & $0(0.0)$ & $3(4.4)$ & \\
\hline Autoimmune & $3(3.3)$ & $00.0)$ & $3(4.4)$ & \\
\hline Unknown & $58(63.7)$ & $16(69.6)$ & $42(61.8)$ & \\
\hline \multicolumn{5}{|l|}{ Number of ASMs } \\
\hline 1 & $7(7.7)$ & $1(4.3)$ & $6(8.8)$ & \\
\hline 2 & $24(26.4)$ & $6(26.1)$ & $18(26.5)$ & \\
\hline 3 & $35(38.5)$ & $10(43.5)$ & $25(36.8)$ & \\
\hline 4 & $14(15.4)$ & $5(21.7)$ & $9(13.2)$ & \\
\hline 5 & $9(9.9)$ & $1(4.3)$ & $8(11.8)$ & \\
\hline 6 & $2(2.2)$ & $0(0.0)$ & $2(2.9)$ & \\
\hline \multicolumn{5}{|l|}{ Concurrent ASMs } \\
\hline Carbamazepine & 23 & 5 & 18 & \\
\hline Clobazam & 9 & 1 & 8 & \\
\hline Gabapentin & 2 & 0 & 2 & \\
\hline Lacosamide & 3 & 1 & 2 & \\
\hline Lamotrigine & 35 & 9 & 26 & \\
\hline Levetiracetam & 54 & 13 & 41 & \\
\hline Oxcarbazepine & 15 & 7 & 8 & \\
\hline Perampanel & 11 & 1 & 10 & \\
\hline Phenobarbital & 12 & 2 & 10 & \\
\hline Phenytoin & 18 & 4 & 14 & \\
\hline Pregabalin & 3 & 0 & 3 & \\
\hline Topiramate & 28 & 11 & 17 & \\
\hline Valproic acid & 32 & 8 & 24 & \\
\hline Vigabatrin & 4 & 1 & 3 & \\
\hline Zonisamide & 24 & 5 & 19 & \\
\hline
\end{tabular}

Continuous variables were presented as median (interquartile range) Categorical variables were presented as $\mathrm{n}(\%)$ 
variants found in dbSNP build 147 were selected for further analysis. The mean read depth of the panel was $300.1 \mathrm{x}$ and $78.6 \%$ coverage of the target region for at least 20 reads.

\section{Statistical analysis}

The $\chi^{2}$ test or Fisher's exact test is applied to test if the genetic polymorphisms are in agreement with HardyWeinberg equilibrium. Those who did not meet HardyWeinberg equilibrium were excluded from further study. The $x^{2}$ test or Fisher's exact test was used where appropriate to assess differences in genotype and allele frequencies between the patients who had a response to ASMs and not achieving seizure-free. Among the patients who did not achieve seizure-free, the same statistical method was applied to assess differences between the patients with a partial response and failure to ASMs. Bonferroni correction was applied to rule out falsepositive associations after multiple comparisons, with $\mathrm{p}<\alpha / \mathrm{n}$ ( $n=$ total number of statistical tests) considered significant when comparing genotypes and alleles with the drug response.

Table 3 Demographic data of patients taking predominant sodium channel blocking antiseizure medications

\begin{tabular}{|c|c|c|c|c|}
\hline & All $(n=82)$ & Response $(n=58)$ & Not seizure-free $(n=24)$ & $p$ \\
\hline Onset age (year) & $17.5(12.8-26.0)$ & $15.0(8.0-19.0)$ & $19.0(15.0-28.0)$ & 0.020 \\
\hline Male & $34(41.5)$ & $24(41.4)$ & $10(41.7)$ & reference \\
\hline Female & $48(58.5)$ & $34(58.6)$ & $14(58.3)$ & 0.981 \\
\hline \multicolumn{5}{|l|}{ Seizure type } \\
\hline Focal & $51(62.2)$ & $34(58.6)$ & $17(70.8)$ & \multirow[t]{3}{*}{0.382} \\
\hline Generalized & $17(20.7)$ & $12(20.7)$ & $5(20.8)$ & \\
\hline Unspecified & $14(17.1)$ & $12(20.7)$ & $2(8.3)$ & \\
\hline \multicolumn{5}{|l|}{ Etiology } \\
\hline Structural & $13(15.9)$ & $8(13.8)$ & $5(20.8)$ & \multirow[t]{5}{*}{0.072} \\
\hline CNS infection & $4(4.9)$ & $3(5.2)$ & $1(4.2)$ & \\
\hline Genetic & $1(1.2)$ & $0(0.0)$ & $1(4.2)$ & \\
\hline Autoimmune & $2(2.4)$ & $0(0.0)$ & $2(8.3)$ & \\
\hline Unknown & $62(75.6)$ & $47(81.0)$ & $15(62.5)$ & \\
\hline \multicolumn{5}{|l|}{ Number of ASMs } \\
\hline 1 & $46(56.1)$ & $40(69.0)$ & $6(25.0)$ & \\
\hline 2 & $23(28.0)$ & $13(22.4)$ & $10(41.7)$ & \\
\hline 3 & $12(14.6)$ & $5(8.6)$ & $7(29.2)$ & \\
\hline 4 & $0(0.0)$ & $0(0.0)$ & $0(0.0)$ & \\
\hline 5 & $1(1.2)$ & $0(0.0)$ & $1(4.2)$ & \\
\hline \multicolumn{5}{|l|}{ Concurrent ASMs } \\
\hline Carbamazepine & 29 & 21 & 8 & \\
\hline Clobazam & 3 & 2 & 1 & \\
\hline Gabapentin & 1 & 0 & 1 & \\
\hline Lacosamide & 2 & 0 & 2 & \\
\hline Lamotrigine & 34 & 24 & 10 & \\
\hline Levetiracetam & 24 & 10 & 14 & \\
\hline Oxcarbazepine & 10 & 6 & 4 & \\
\hline Perampanel & 1 & 0 & 1 & \\
\hline Phenobarbital & 1 & 0 & 1 & \\
\hline Phenytoin & 27 & 18 & 9 & \\
\hline Vigabatrin & 1 & 0 & 1 & \\
\hline
\end{tabular}

Continuous variables were presented as median (interquartile range) Categorical variables were presented as $\mathrm{n}(\%)$

Abbreviations: CNS central nervous system, ASM antiseizure medication 


\section{Results}

\section{Demographic characteristics}

During the study period, 214 Taiwanese patients who took SCN-ASMs were enrolled in the study. One patient was excluded due to noncompliance and 13 patients had an unknown response to SCN-ASMs. The remaining 200 patients were then analyzed.

The clinical characteristics of the patients taking SCN-ASMs are shown in Table 1. One hundred and nine $(54.5 \%)$ patients were classified as having a response to SCN-ASMs. Focal epilepsy was the most prevalent seizure type $(60.5 \%)$ followed by generalized (25.5\%) and unclassified (14.0\%). Most of the patients had an unknown etiology (71.0\%). The known etiologies included structural lesions (19.0\%), central nervous system (CNS) infections (6.0\%), genetic (2.0\%), and autoimmune $(2.0 \%)$. The age at onset was significantly older in the patients with a response to SCNASMs. The clinical characteristics of patients not achieving seizure-free to SCN-ASMs $(n=91)$ were shown in Table 2, which were categorized into having partial response and failure.

Table 4 Demographic data of patients with partial response and failure to predominant sodium channel blocking antiseizure medications

\begin{tabular}{|c|c|c|c|c|}
\hline & All $(n=24)$ & Partial response $(n=5)$ & Failure $(n=19)$ & $p$ \\
\hline Onset age & $15.0(6.0-19.5)$ & $6.5(1.3-14.0)$ & $16.0(10.0-21.0)$ & 0.586 \\
\hline Male & $10(41.7)$ & $2(40.0)$ & $8(42.1)$ & reference \\
\hline Female & $14(58.3)$ & $3(60.0)$ & $11(57.9)$ & 1.000 \\
\hline \multicolumn{5}{|l|}{ Seizure type } \\
\hline Focal & $17(70.8)$ & $2(40.0)$ & $15(78.9)$ & \multirow[t]{3}{*}{0.224} \\
\hline Generalized & $5(20.8)$ & $2(40.0)$ & $3(15.8)$ & \\
\hline Unspecified & $2(8.3)$ & $1(20.0)$ & $1(5.3)$ & \\
\hline \multicolumn{5}{|l|}{ Etiology } \\
\hline Structural & $5(20.8)$ & $1(20.0)$ & $4(21.1)$ & \multirow[t]{5}{*}{0.331} \\
\hline CNS infection & $1(4.2)$ & $1(20.0)$ & $0(0.0)$ & \\
\hline Genetic & $1(4.2)$ & $0(0.0)$ & $1(5.3)$ & \\
\hline Autoimmune & $2(8.3)$ & $0(0.0)$ & $2(10.5)$ & \\
\hline Unknown & $15(62.5)$ & $3(60.0)$ & $12(63.2)$ & \\
\hline \multicolumn{5}{|l|}{ Number of ASMs } \\
\hline 1 & $6(25.0)$ & $1(20.0)$ & $5(26.3)$ & \multirow[t]{5}{*}{0.965} \\
\hline 2 & $10(41.7)$ & $2(40.0)$ & $8(42.1)$ & \\
\hline 3 & $7(29.2)$ & $2(40.0)$ & $5(26.3)$ & \\
\hline 4 & $0(0.0)$ & $0(0.0)$ & $0(0.0)$ & \\
\hline 5 & $1(5.9)$ & $0(0.0)$ & $1(5.3)$ & \\
\hline \multicolumn{5}{|l|}{ Concurrent ASMs } \\
\hline Carbamazepine & 8 & 2 & 6 & \\
\hline Clobazam & 1 & 0 & 1 & \\
\hline Gabapentin & 1 & 0 & 1 & \\
\hline Lacosamide & 2 & 1 & 1 & \\
\hline Lamotrigine & 10 & 1 & 9 & \\
\hline Levetiracetam & 14 & 3 & 11 & \\
\hline Oxcarbazepine & 4 & 2 & 2 & \\
\hline Perampanel & 1 & 0 & 1 & \\
\hline Phenobarbital & 1 & 0 & 1 & \\
\hline Phenytoin & 9 & 2 & 7 & \\
\hline Vigabatrin & 1 & 0 & 1 & \\
\hline
\end{tabular}

Continuous variables were presented as median (interquartile range) Categorical variables were presented as $\mathrm{n}(\%)$

Abbreviations: CNS central nervous system, ASM antiseizure medication 
After excluding patients using broad-spectrum mechanism ASMs (valproate acid, topiramate, and zonisamide), 82 patients were classified into the predominant SCN-ASMs subgroup. The clinical characteristics of these 82 patients are presented in Table 3. Among these patients, $58(70.7 \%)$ were responsive to predominant SCN-ASMs. The focal seizure was the most prevalent seizure type $(62.2 \%)$ followed by generalized (20.7\%) and unclassified (17.1\%). With regards to the etiology, $15.9 \%$ had structural lesions, $4.9 \%$ had CNS infections, $1.2 \%$ had a genetic etiology, $2.4 \%$ had an autoimmune etiology, and $75.6 \%$ were unknown. In this group, the age at onset was significantly younger in the patients who responded to predominant SCN-ASMs. The clinical characteristics of patients not achieving seizure-free to predominant SCN-ASMs $(n=24)$ were shown in Table 4 , which were categorized into having partial response and failure.

\section{Associations between SCN gene SNPs and ASM responsiveness}

Using the gene panel, we detected three SNPs in SCN1A (rs121918808, rs200176684, and rs201985242), seven in SCN1B (rs55742440, rs3746255, rs67486287, rs67701503, rs2305748, rs369032304, and rs72558026), six in SCN2A (rs2060198, rs17183814, rs138497939, rs185590667, rs199925238, and rs186154973), and five in SCN9A (rs9646771, rs3750904, rs199756028, rs200613417, and rs200956485). With 21 SNPs analyzed, the overall significance level is set to be 0.0024 or less after applying the Bonferroni correction.

Table 5 lists the characteristic of the SNPs from SCN1A, SCN1B, SCN2A, and SCN9A genes in 200 patients taking SCN-ASMs. Only allele $C$ of rs55742440 in SCN1B (OR: 0.466; 95\% CI: 0.287-0.754; $p=0.0016$ ) was statistically significantly associated with not achieving seizure-free with SCN-ASMs. We further examined whether specific SCN-ASM having a higher risk of

Table 5 Allele and genotype distribution of patients with response and not achieving seizure free to sodium channel blocking antiseizure medications

\begin{tabular}{|c|c|c|c|c|c|c|c|c|c|c|c|c|}
\hline \multirow[t]{3}{*}{ Gene } & \multirow[t]{3}{*}{ SNPs } & \multicolumn{8}{|c|}{ Genotypes } & \multicolumn{3}{|l|}{ Alleles } \\
\hline & & \multirow[b]{2}{*}{$A / B$} & \multicolumn{3}{|c|}{ Response $(n=109)$} & \multicolumn{3}{|c|}{ Not seizure-free $(n=91)$} & \multirow[b]{2}{*}{$p$} & \multirow{2}{*}{$\begin{array}{l}\text { Response A: } \\
\text { B; not } \\
\text { seizure-free } \\
\text { A:B }\end{array}$} & \multirow{2}{*}{$\begin{array}{l}\text { Odds ratio } \\
(95 \% \mathrm{Cl})\end{array}$} & \multirow[t]{2}{*}{$P^{*}$} \\
\hline & & & $\overline{A A}$ & $A B$ & BB & $\overline{A A}$ & $A B$ & BB & & & & \\
\hline \multirow[t]{3}{*}{ SCN1A } & rs121918808 & $\mathrm{C} / \mathrm{T}$ & 108 & 1 & 0 & 90 & 1 & 0 & 1.0000 & $217: 1 ; 181: 1$ & $0.834(0.052-13.430)$ & 1.0000 \\
\hline & rs200176684 & $\mathrm{C} / \mathrm{T}$ & 108 & 0 & 1 & 91 & 0 & 0 & 1.0000 & $216: 2 ; 182: 0$ & & 0.5028 \\
\hline & rs201985242 & $\mathrm{G} / \mathrm{C}$ & 109 & 0 & 0 & 90 & 1 & 0 & 0.7017 & 218:0; 181:1 & & 0.4550 \\
\hline \multirow[t]{7}{*}{ SCN1B } & rs55742440 & $\mathrm{T} / \mathrm{C}$ & 77 & 29 & 3 & 47 & 35 & 9 & 0.0097 & $183: 35 ; 129: 53$ & $0.466(0.287-0.754)$ & 0.0016 \\
\hline & rs3746255 & $\mathrm{C} / \mathrm{T}$ & 104 & 4 & 1 & 85 & 6 & 0 & 0.5169 & $212: 6 ; 176: 6$ & $0.830(0.263-2.620)$ & 0.7773 \\
\hline & rs67486287 & $\mathrm{G} / \mathrm{C}$ & 80 & 27 & 2 & 57 & 28 & 6 & 0.1140 & $187: 31 ; 142: 40$ & $0.589(0.351-0.987)$ & 0.0431 \\
\hline & rs67701503 & $C / A$ & 80 & 27 & 2 & 57 & 28 & 6 & 0.1140 & $187: 31 ; 142: 40$ & $0.589(0.351-0.987)$ & 0.0431 \\
\hline & rs2305748 & $\mathrm{C} / \mathrm{T}$ & 80 & 27 & 2 & 57 & 28 & 6 & 0.1140 & $187: 31 ; 142: 40$ & $0.589(0.351-0.987)$ & 0.0431 \\
\hline & rs369032304 & $\mathrm{G} / \mathrm{A}$ & 109 & 0 & 0 & 90 & 1 & 0 & 0.4550 & 218:0; 181:1 & & 0.4550 \\
\hline & rs72558026 & $\mathrm{G} / \mathrm{A}$ & 108 & 1 & 0 & 86 & 5 & 0 & 0.0941 & $217: 1 ; 177: 5$ & $0.163(0.019-1.409)$ & 0.0965 \\
\hline \multirow[t]{6}{*}{ SCN2A } & rs2060198 & T/A & 103 & 5 & 1 & 87 & 3 & 1 & 0.8656 & $211: 7 ; 177: 5$ & $1.174(0.366-3.765)$ & 0.7913 \\
\hline & rs17183814 & $\mathrm{G} / \mathrm{A}$ & 76 & 30 & 3 & 62 & 27 & 2 & 0.9169 & $182: 36 ; 151: 31$ & $0.964(0.569-1.631)$ & 0.8875 \\
\hline & rs138497939 & $\mathrm{A} / \mathrm{C}$ & 107 & 2 & 0 & 91 & 0 & 0 & 0.5016 & $216: 2 ; 182: 0$ & & 0.5028 \\
\hline & rs185590667 & $\mathrm{T} / \mathrm{C}$ & 108 & 1 & 0 & 91 & 0 & 0 & 1.0000 & $217: 1 ; 182: 0$ & & 1.0000 \\
\hline & rs 199925238 & $\mathrm{G} / \mathrm{A}$ & 108 & 1 & 0 & 91 & 0 & 0 & 1.0000 & $217: 1 ; 182: 0$ & & 1.0000 \\
\hline & rs186154973 & $\mathrm{G} / \mathrm{A}$ & 108 & 1 & 0 & 91 & 0 & 0 & 1.0000 & $217: 1 ; 182: 0$ & & 1.0000 \\
\hline \multirow[t]{5}{*}{ SCN9A } & rs9646771 & $\mathrm{T} / \mathrm{C}$ & 108 & 1 & 0 & 91 & 0 & 0 & 1.0000 & $217: 1 ; 182: 0$ & & 1.0000 \\
\hline & rs3750904 & $\mathrm{T} / \mathrm{C}$ & 78 & 26 & 5 & 69 & 20 & 2 & 0.6430 & $182: 36 ; 158: 24$ & $1.302(0.745-2.277)$ & 0.3537 \\
\hline & rs199756028 & $\mathrm{G} / \mathrm{A}$ & 108 & 1 & 0 & 91 & 0 & 0 & 1.0000 & $217: 1 ; 182: 0$ & & 1.0000 \\
\hline & rs200613417 & $\mathrm{A} / \mathrm{T}$ & 109 & 0 & 0 & 90 & 1 & 0 & 0.4550 & 218:0; 181:1 & & 0.4550 \\
\hline & rs200956485 & $\mathrm{G} / \mathrm{A}$ & 108 & 1 & 0 & 91 & 0 & 0 & 1.0000 & $217: 1 ; 182: 0$ & & 1.0000 \\
\hline
\end{tabular}


not achieving seizure-free, Fisher's exact test was performed on patients with rs55742440T $>C$, to find which last SCN-ASM is related to not achieving seizure-free and Bonferroni correction was applied after multiple comparisons. With a total of eight SCN-ASMs and the corrected $p$-value was set at 0.00625 after Bonferroni correction, no particular ASM was significantly associated with not achieving seizure-free (Supplement Table).

Table 6 lists the associations between the SNPs and the 91 patients with a partial response or failure to SCNASMs, none were found to have significant associations. Table 7 lists the associations between the SNPs and the response of 82 patients taking predominant SCN-ASMs and Table 8 lists the associations between the SNPs and the 24 patients with partial response or failure to predominant SCN-ASMs. No SNPs were found to be associated with the drug responsiveness for patients taking predominant SCN-ASMs.

\section{Discussion}

In this study, the C allele of rs55742440 in SCN1B was statistically significantly associated with not achieving seizure-free with SCN-ASMs among Taiwanese patients. This finding is consistent with the new perspective that the beta subunit of SCN, which is encoded by $S C N 1 B$ [28], may influence the function of SCNs.

Voltage-gated SCNs are composed of alpha and beta subunits [29]. The alpha subunits form the functional pore of the channel, and the beta subunits serve to modulate the channel's biophysical properties [30]. Mutations of the alpha subunits have been shown to cause Dravet syndrome and generalized epilepsy with febrile seizures plus [10], which could also be caused by mutations in the beta subunits $[10,31,32]$. No previous study has reported an association between SCN1B and ASM responsiveness in humans, although animal models have shown that mutations of SCN1B can reduce sensitivity to ASMs [33-35]. Our findings suggest that SNPs in

Table 6 Allele and genotype distribution of the patients with partial response and failure to sodium channel blocking antiseizure medications

\begin{tabular}{|c|c|c|c|c|c|c|c|c|c|c|c|c|}
\hline \multirow[t]{3}{*}{ Gene } & \multirow[t]{3}{*}{ SNPs } & \multicolumn{8}{|c|}{ Genotypes } & \multicolumn{3}{|l|}{ Alleles } \\
\hline & & \multirow[b]{2}{*}{$A / B$} & \multicolumn{3}{|c|}{ Partial response $(n=23)$} & \multicolumn{3}{|c|}{ Failure $(n=68)$} & \multirow[b]{2}{*}{$p$} & \multirow{2}{*}{$\begin{array}{l}\text { Partial } \\
\text { response A: } \\
\text { B; failure A: } \\
\text { B }\end{array}$} & \multirow{2}{*}{$\begin{array}{l}\text { Odds ratio } \\
(95 \% \mathrm{Cl})\end{array}$} & \multirow[t]{2}{*}{$P^{*}$} \\
\hline & & & $\overline{A A}$ & $A B$ & BB & $\overline{A A}$ & $A B$ & BB & & & & \\
\hline \multirow[t]{3}{*}{ SCNIA } & rs121918808 & $\mathrm{C} / \mathrm{T}$ & 22 & 1 & 0 & 68 & 0 & 0 & 0.2527 & $45: 1 ; 136: 0$ & & 0.2527 \\
\hline & rs200176684 & $\mathrm{C} / \mathrm{T}$ & 23 & 0 & 0 & 68 & 0 & 0 & 1.0000 & $46: 0 ; 136: 0$ & & 1.0000 \\
\hline & rs201985242 & $\mathrm{G} / \mathrm{C}$ & 23 & 0 & 0 & 67 & 1 & 0 & 1.0000 & $46: 0 ; 135: 1$ & & 1.0000 \\
\hline \multirow[t]{7}{*}{ SCN1B } & rs55742440 & $\mathrm{T} / \mathrm{C}$ & 12 & 9 & 2 & 35 & 26 & 7 & 0.9753 & $33: 13 ; 96: 40$ & $0.946(0.451-1.982)$ & 0.8875 \\
\hline & rs3746255 & $\mathrm{C} / \mathrm{T}$ & 21 & 2 & 0 & 64 & 4 & 0 & 0.6403 & $44: 2 ; 132: 4$ & $1.500(0.266-8.473)$ & 0.4743 \\
\hline & rs67486287 & $\mathrm{G} / \mathrm{C}$ & 13 & 9 & 1 & 44 & 19 & 5 & 0.6175 & $35: 11 ; 107: 29$ & $1.160(0.525-2.560)$ & 0.7184 \\
\hline & rs67701503 & $\mathrm{C} / \mathrm{A}$ & 13 & 9 & 1 & 44 & 19 & 5 & 0.6175 & $35: 11 ; 107: 29$ & $1.160(0.525-2.560)$ & 0.7184 \\
\hline & rs2305748 & $\mathrm{C} / \mathrm{T}$ & 13 & 9 & 1 & 44 & 19 & 5 & 0.6175 & $35: 11 ; 107: 29$ & $1.160(0.525-2.560)$ & 0.7184 \\
\hline & rs369032304 & $\mathrm{G} / \mathrm{A}$ & 23 & 0 & 0 & 67 & 1 & 0 & 1.0000 & $46: 0 ; 135: 1$ & & 1.0000 \\
\hline & rs72558026 & $\mathrm{G} / \mathrm{A}$ & 22 & 1 & 0 & 64 & 4 & 0 & 1.0000 & $45: 1 ; 132: 4$ & $0.733(0.080-6.734)$ & 1.0000 \\
\hline \multirow[t]{6}{*}{ SCN2A } & rs2060198 & $\mathrm{T} / \mathrm{A}$ & 23 & 0 & 0 & 64 & 3 & 1 & 0.6765 & $46: 0 ; 131: 5$ & & 0.3321 \\
\hline & rs17183814 & $\mathrm{G} / \mathrm{A}$ & 17 & 6 & 0 & 45 & 21 & 2 & 0.8849 & 40:6; 111:25 & $0.666(0.255-1.742)$ & 0.4061 \\
\hline & rs138497939 & $\mathrm{A} / \mathrm{C}$ & 23 & 0 & 0 & 68 & 0 & 0 & 1.0000 & $46: 0 ; 136: 0$ & & 1.0000 \\
\hline & rs185590667 & $\mathrm{T} / \mathrm{C}$ & 23 & 0 & 0 & 68 & 0 & 0 & 1.0000 & $46: 0 ; 136: 0$ & & 1.0000 \\
\hline & rs199925238 & $\mathrm{G} / \mathrm{A}$ & 21 & 2 & 0 & 63 & 5 & 0 & 1.0000 & $44: 2 ; 131: 5$ & & 1.0000 \\
\hline & rs186154973 & $G / A$ & 23 & 0 & 0 & 68 & 0 & 0 & 1.0000 & $46: 0 ; 136: 0$ & & 1.0000 \\
\hline \multirow[t]{5}{*}{ SCN9A } & rs9646771 & $\mathrm{T} / \mathrm{C}$ & 13 & 7 & 3 & 48 & 12 & 8 & 0.3272 & $33: 13 ; 108: 28$ & $1.520(0.707-3.264)$ & 0.2814 \\
\hline & rs3750904 & $\mathrm{T} / \mathrm{C}$ & 18 & 5 & 0 & 51 & 15 & 2 & 1.0000 & $41: 5 ; 117: 19$ & $0.751(0.263-2.140)$ & 0.5902 \\
\hline & rs199756028 & $\mathrm{G} / \mathrm{A}$ & 23 & 0 & 0 & 68 & 0 & 0 & 1.0000 & $46: 0 ; 136: 0$ & & 1.0000 \\
\hline & rs200613417 & $\mathrm{A} / \mathrm{T}$ & 23 & 0 & 0 & 67 & 1 & 0 & 1.0000 & $46: 0 ; 135: 1$ & & 1.0000 \\
\hline & rs200956485 & $\mathrm{G} / \mathrm{A}$ & 23 & 0 & 0 & 68 & 0 & 0 & 1.0000 & $46: 0 ; 136: 0$ & & 1.0000 \\
\hline
\end{tabular}


Table 7 Allele and genotype distribution of patients with response and not achieving seizure free to predominant sodium channel blocking antiseizure medications

\begin{tabular}{|c|c|c|c|c|c|c|c|c|c|c|c|c|}
\hline \multirow[t]{3}{*}{ Gene } & \multirow[t]{3}{*}{ SNPs } & \multicolumn{8}{|c|}{ Genotypes } & \multicolumn{3}{|l|}{ Alleles } \\
\hline & & \multirow[b]{2}{*}{$A / B$} & \multicolumn{3}{|c|}{ Response $(n=58)$} & \multicolumn{3}{|c|}{ Not seizure-free $(n=24)$} & \multirow[b]{2}{*}{$p$} & \multirow{2}{*}{$\begin{array}{l}\text { Response } \\
A: B ; \text { not } \\
\text { seizure- } \\
\text { free } A: B\end{array}$} & \multirow{2}{*}{$\begin{array}{l}\text { Odds ratio }(95 \% \\
\text { Cl) }\end{array}$} & \multirow[t]{2}{*}{$P^{*}$} \\
\hline & & & AA & $A B$ & BB & $\mathrm{AA}$ & $A B$ & BB & & & & \\
\hline \multirow[t]{3}{*}{ SCN1A } & rs121918808 & $C / T$ & 57 & 1 & 0 & 24 & 0 & 0 & 1.0000 & $115: 1 ; 48: 0$ & & 1.0000 \\
\hline & rs 200176684 & $C / T$ & 57 & 0 & 1 & 24 & 0 & 0 & 1.0000 & $114: 2 ; 48: 0$ & & 0.5834 \\
\hline & rs201985242 & $\mathrm{G} / \mathrm{C}$ & 58 & 0 & 0 & 24 & 0 & 0 & 1.0000 & $116: 0 ; 48: 0$ & & 1.0000 \\
\hline \multirow[t]{7}{*}{ SCN1B } & rs55742440 & $\mathrm{T} / \mathrm{C}$ & 40 & 17 & 1 & 12 & 8 & 4 & 0.0310 & $97: 19 ; 32: 16$ & $0.392(0.180-0.851)$ & 0.0159 \\
\hline & rs3746255 & $C / T$ & 57 & 1 & 0 & 22 & 2 & 0 & 0.2036 & $115: 1 ; 46: 2$ & $0.2(0.017-2.260)$ & 0.2052 \\
\hline & rs67486287 & $\mathrm{G} / \mathrm{C}$ & 41 & 16 & 1 & 17 & 5 & 2 & 0.3292 & $98: 18 ; 39: 9$ & $0.796(0.330-1.923)$ & 0.6101 \\
\hline & rs67701503 & $C / A$ & 41 & 16 & 1 & 17 & 5 & 2 & 0.3292 & $98: 18 ; 39: 9$ & $0.796(0.330-1.923)$ & 0.6101 \\
\hline & rs2305748 & $C / T$ & 41 & 16 & 1 & 17 & 5 & 2 & 0.3292 & $98: 18 ; 39: 9$ & $0.796(0.330-1.923)$ & 0.6101 \\
\hline & rs369032304 & $\mathrm{G} / \mathrm{A}$ & 58 & 0 & 0 & 24 & 0 & 0 & 1.0000 & $116: 0 ; 48: 0$ & & 1.0000 \\
\hline & rs72558026 & $\mathrm{G} / \mathrm{A}$ & 57 & 1 & 0 & 20 & 4 & 0 & 0.0241 & $115: 1 ; 44: 4$ & $0.096(0.010-0.880)$ & 0.0261 \\
\hline \multirow[t]{6}{*}{ SCN2A } & rs2060198 & T/A & 54 & 4 & 0 & 24 & 0 & 0 & 0.3158 & $112: 4 ; 48: 0$ & & 0.3222 \\
\hline & rs17183814 & $\mathrm{G} / \mathrm{A}$ & 41 & 14 & 3 & 14 & 9 & 1 & 0.4452 & $96: 20 ; 37: 11$ & $0.701(0.306-1.603)$ & 0.5110 \\
\hline & rs138497939 & $\mathrm{A} / \mathrm{C}$ & 57 & 1 & 0 & 24 & 0 & 0 & 1.0000 & $115: 1 ; 48: 0$ & & 1.0000 \\
\hline & rs185590667 & $\mathrm{T} / \mathrm{C}$ & 57 & 1 & 0 & 24 & 0 & 0 & 1.0000 & $115: 1 ; 48: 0$ & & 1.0000 \\
\hline & rs 199925238 & $\mathrm{G} / \mathrm{A}$ & 54 & 4 & 0 & 21 & 3 & 0 & 0.4418 & $112: 4 ; 45: 3$ & $0.536(0.115-2.490)$ & 0.6746 \\
\hline & rs186154973 & $\mathrm{G} / \mathrm{A}$ & 57 & 1 & 0 & 24 & 0 & 0 & 1.0000 & $115: 1 ; 48: 0$ & & 1.0000 \\
\hline \multirow[t]{5}{*}{ SCN9A } & rs9646771 & $\mathrm{T} / \mathrm{C}$ & 39 & 12 & 7 & 14 & 5 & 5 & 0.5625 & $90: 26 ; 33: 15$ & $0.636(0.300-1.346)$ & 0.2351 \\
\hline & rs3750904 & $\mathrm{T} / \mathrm{C}$ & 44 & 11 & 3 & 18 & 6 & 0 & 0.6353 & $99: 17 ; 42: 6$ & $1.202(0.433-3.261)$ & 0.7184 \\
\hline & rs199756028 & $\mathrm{G} / \mathrm{A}$ & 57 & 1 & 0 & 24 & 0 & 0 & 1.0000 & $115: 1 ; 48: 0$ & & 1.0000 \\
\hline & rs200613417 & $\mathrm{A} / \mathrm{T}$ & 58 & 0 & 0 & 23 & 1 & 0 & 0.2926 & $116: 0 ; 47: 1$ & & 0.2927 \\
\hline & rs200956485 & $\mathrm{G} / \mathrm{A}$ & 57 & 1 & 0 & 24 & 0 & 0 & 1.0000 & $115: 1 ; 48: 0$ & & 1.0000 \\
\hline
\end{tabular}

Abbreviations: $\mathrm{Cl}$ confidence interval, SNP single nucleotide polymorphism

* The significance level is set to be 0.0024 or less using Bonferroni correction

$S C N 1 B$ could cause functional alterations of SCNs, thereby affecting the responsiveness to ASMs.

In the current study, rs55742440T $>C$ in $S C N 1 B$ was associated with not achieving seizure-free with SCNASMs. The rs55742440T >C SNP causes a missense change of the amino acid (p.Leu210Pro) located in the intracellular C-terminal domain of $S C N 1 B$. According to the gnomAD database [36], it is prevalent across all ethnicities $(\mathrm{MAF}=0.38)$, not only in the East Asian population $(\mathrm{MAF}=0.27)$ where this study cohort origin. Therefore, this SNP is unlikely to be pathogenic for epilepsy based on the ACMG guideline. Nevertheless, the variant has been reported in patients with Brugada syndrome and arrhythmogenic right ventricular cardiomyopathy [37-39], it was also demonstrated to reduce sodium channel current in cardiomyocytes together with another missense variant [39]. It is possible that the SCN1B variant modifies the function of alpha subunits of voltage-gated sodium channels and makes it less responsive to SCN-ASMs via a currently unknown mechanism. Taken together, rs55742440 may affect sodium channel function in the heart and brain as well as responsiveness to medications targeting sodium channels. Further functional investigations are warranted to elucidate this issue.

When the analysis was done for the more restricted predominant SCN-ASMs group, no association of drug responsiveness was found for $\mathrm{rs} 55742440 \mathrm{~T}>\mathrm{C}$ in $S C N 1 B$. This could have resulted from the decrease in patient numbers in the predominant SCN-ASMs group. We also observed a difference in the age at onset between the responsiveness to $\mathrm{SCN}-\mathrm{ASM}$ and the more restricted predominant SCN-ASMs. In previous studies, the age at onset was not related to the responsiveness to ASMs [2, 40-42]. This could be caused by bias due to the relatively small sample size in our study, which is crucial to genetic association studies [43].

Our study was limited by the relatively small sample size and the need for multiple statistical tests for association studies. The sample size is a crucial component of 
Table 8 Allele and genotype distribution of patients with partial response and failure to predominant sodium channel blocking antiseizure medications

\begin{tabular}{|c|c|c|c|c|c|c|c|c|c|c|c|c|}
\hline \multirow[t]{3}{*}{ Gene } & \multirow[t]{3}{*}{ SNPs } & \multicolumn{8}{|c|}{ Genotypes } & \multicolumn{3}{|l|}{ Alleles } \\
\hline & & \multirow[b]{2}{*}{$A / B$} & \multicolumn{3}{|c|}{ Partial response $(n=5)$} & \multicolumn{3}{|c|}{ Failure $(n=19)$} & \multirow[b]{2}{*}{$p$} & \multirow{2}{*}{$\begin{array}{l}\text { Partial } \\
\text { response } \\
A: B ; \\
\text { failure A: } \\
B\end{array}$} & \multirow{2}{*}{$\begin{array}{l}\text { Odds ratio }(95 \% \\
\text { Cl) }\end{array}$} & \multirow[t]{2}{*}{$p^{*}$} \\
\hline & & & $\overline{\mathrm{AA}}$ & $A B$ & BB & $\overline{A A}$ & $A B$ & BB & & & & \\
\hline \multirow[t]{3}{*}{ SCN1A } & rs121918808 & $\mathrm{C} / \mathrm{T}$ & 5 & 0 & 0 & 19 & 0 & 0 & 1.0000 & $10: 0 ; 38: 0$ & & 1.0000 \\
\hline & rs200176684 & $\mathrm{C} / \mathrm{T}$ & 5 & 0 & 0 & 19 & 0 & 0 & 1.0000 & $10: 0 ; 38: 0$ & & 1.0000 \\
\hline & rs201985242 & $\mathrm{G} / \mathrm{C}$ & 5 & 0 & 0 & 19 & 0 & 0 & 1.0000 & 10:0; $38: 0$ & & 1.0000 \\
\hline \multirow[t]{7}{*}{ SCNIB } & rs55742440 & $\mathrm{T} / \mathrm{C}$ & 2 & 2 & 1 & 10 & 6 & 3 & 1.0000 & $6: 4 ; 26: 12$ & $1.444(0.342-6.086)$ & 0.7118 \\
\hline & rs3746255 & $\mathrm{C} / \mathrm{T}$ & 5 & 0 & 0 & 17 & 2 & 0 & 1.0000 & $10: 0 ; 36: 2$ & & 1.0000 \\
\hline & rs67486287 & $\mathrm{G} / \mathrm{C}$ & 2 & 3 & 0 & 15 & 2 & 2 & 0.0785 & $7: 3 ; 32: 6$ & $2.286(0.457-11.426)$ & 0.3698 \\
\hline & rs67701503 & $C / A$ & 2 & 3 & 0 & 15 & 2 & 2 & 0.0785 & $7: 3 ; 32: 6$ & $2.286(0.457-11.426)$ & 0.3698 \\
\hline & rs2305748 & $\mathrm{C} / \mathrm{T}$ & 2 & 3 & 0 & 15 & 2 & 2 & 0.0785 & $7: 3 ; 32: 6$ & $2.286(0.457-11.426)$ & 0.3698 \\
\hline & rs369032304 & $\mathrm{G} / \mathrm{A}$ & 5 & 0 & 0 & 19 & 0 & 0 & 1.0000 & 10:0; 38:0 & & 1.0000 \\
\hline & rs72558026 & $\mathrm{G} / \mathrm{A}$ & 5 & 0 & 0 & 15 & 4 & 0 & 0.5440 & $10: 0 ; 34: 4$ & & 0.5665 \\
\hline \multirow[t]{6}{*}{ SCN2A } & rs2060198 & $\mathrm{T} / \mathrm{A}$ & 5 & 0 & 0 & 19 & 0 & 0 & 1.0000 & 10:0; 38:0 & & 1.0000 \\
\hline & rs17183814 & $\mathrm{G} / \mathrm{A}$ & 2 & 3 & 0 & 12 & 6 & 1 & 0.4797 & $7: 3 ; 30: 8$ & $1.607(0.337-7.658)$ & 0.6754 \\
\hline & rs138497939 & $\mathrm{A} / \mathrm{C}$ & 5 & 0 & 0 & 19 & 0 & 0 & 1.0000 & 10:0; 38:0 & & 1.0000 \\
\hline & rs185590667 & $\mathrm{T} / \mathrm{C}$ & 5 & 0 & 0 & 19 & 0 & 0 & 1.0000 & 10:0; 38:0 & & 1.0000 \\
\hline & rs199925238 & $\mathrm{G} / \mathrm{A}$ & 4 & 1 & 0 & 17 & 2 & 0 & 0.5212 & $9: 1 ; 36: 2$ & $2.000(0.163-24.590)$ & 1.0000 \\
\hline & rs186154973 & $\mathrm{G} / \mathrm{A}$ & 5 & 0 & 0 & 19 & 0 & 0 & 1.0000 & 10:0; 38:0 & & 1.0000 \\
\hline \multirow[t]{5}{*}{ SCN9A } & rs9646771 & $\mathrm{T} / \mathrm{C}$ & 0 & 3 & 2 & 14 & 2 & 3 & 0.0092 & $3: 7 ; 30: 8$ & $8.75(1.836-41.693)$ & 0.0059 \\
\hline & rs3750904 & $\mathrm{T} / \mathrm{C}$ & 4 & 1 & 0 & 14 & 5 & 0 & 1.0000 & $9: 1 ; 33: 5$ & $0.733(0.076-7.099)$ & 1.0000 \\
\hline & rs199756028 & $\mathrm{G} / \mathrm{A}$ & 5 & 0 & 0 & 19 & 0 & 0 & 1.0000 & 10:0; 38:0 & & 1.0000 \\
\hline & rs200613417 & $A / T$ & 5 & 0 & 0 & 18 & 1 & 0 & 1.0000 & $10: 0 ; 37: 1$ & & 1.0000 \\
\hline & rs200956485 & $\mathrm{G} / \mathrm{A}$ & 5 & 0 & 0 & 19 & 0 & 0 & 1.0000 & $10: 0 ; 38: 0$ & & 1.0000 \\
\hline
\end{tabular}

the power to detect a causal variant in the genetic association study [43]. In practice, however, the sample size was limited by the amount of well-characterized clinical samples and the costs of sequencing. With more delicate sample characterizing, the less sample size one got. As in our case, the sample size dropped as we changed the response of the ASM to a more restricted "predominant SCN-ASMs", which resulted in a loss of power and failure to detect any significant signals. A potential solution to increase the sample size would be performing a metaanalysis $[21,44]$ or international research collaboration, such as the EpiPGX consortium [45]. Although the costs of sequencing are dropping due to recent advancements in genetic sequencing technology, the price is still too expensive for many researchers. Even with affordable prices, the advanced sequencing technique generates an even greater amount of data. More genetic data would accompany multiple statistical tests that could increase false-positive results [46]. To counter this problem, multiple test correction was applied and the traditional
Bonferroni correction may be overly conservative that may not be able to find a significant association [47]. New statistical methods for genetic association studies have been developed [48] and, in the future, we might have better tools to process these immense amounts of data to gain a better insight into the association between genotypes and clinical phenotypes.

We only focus on the variation of SCN genes, but other mechanisms may also contribute to drug resistance epilepsy, including drug transporters and proteins involved in the metabolism of ASMs. The efflux transporters at the endothelial cells of the blood-brain barrier (BBB) may hamper the ASMs' ability to enter the central nervous system and decrease their concentration at the epileptogenic tissues [4]. ATP binding cassette (ABC) transporter superfamily is the major efflux transporter on the BBB that may limit the access of ASMs to the brain [49] but various studies had conflicting results about the association of genetic polymorphism of $\mathrm{ABC}$ transporter genes and the responsiveness of ASM [50- 
52]. The solute carrier (SLC) protein is another transporter protein that is responsible for drug transport in the brain and its polymorphisms have functional significance in terms of the pharmacokinetics of many drugs [53]. Currently, no studies focus on the association of the polymorphism of SLC proteins and the responsiveness of ASMs. Drug-metabolizing genes are polymorphic and can influence the biotransformation of many drugs [54]. Enzyme variants may alter the responsiveness of the ASMs, which makes them ineffective. One Taiwanese study revealed the polymorphism of EPHX was related to a higher dose of carbamazepine for seizure control [55]. Another Chinese study showed the polymorphism of UGT was associated with a higher dose of oxcarbazepine in controlling seizures [56]. The magnitude of the factors influencing the responsiveness of ASM is great, a larger population study and newer methods will be needed to explore this complex interaction.

The response of an ASM is currently unpredictable and usually requires trial at the cost of ongoing seizures or the occurrence of adverse reactions. The pursuit of predicting the responsiveness to ASMs using genetic information, such as SNPs, has not been very successful $[57,58]$, and conflicting results have been observed in gene association studies [12, 13, 15, $16,18,59,60]$. The recent development of polygenic risk scores could be a promising method to unravel the complex relationship between genetic background and drug responsiveness [61, 62]. A recent large whole-exome sequencing study found that rare coding damaging variants were marginally enriched in genes involved in the pharmacokinetics of valproic acid in patients resistant to valproic acid [25]. This is similar to the findings in the present study, in that coding variants of SCN genes (particularly SCN1B) affected the responsiveness to SCN-ASMs. The predictive ability of these findings still needs to be replicated in different study cohorts.

\section{Conclusion}

We identified rs55742440T $>$ C in SCN1B was associated with not achieving seizure-free with SCN-ASMs. although the beta subunits of the SCN were considered as an auxiliary component, our finding supports the concept that the beta subunit could influence the function of the SCN. Based on this finding, further function test about the beta subunit and this SNP is needed to explore the role of rs55742440 in SCN1B.

\section{Abbreviations}

ASM: Antiseizure medication; SCN: Sodium channel; SNP: Single-nucleotide polymorphisms

\section{Supplementary Information}

The online version contains supplementary material available at https://doi. org/10.1186/s12883-021-02395-2.

Additional file 1: Supplement Table. The response of individual sodium channel blocking antiseizure medications in patients with rs55742440T > C.

\section{Acknowledgements}

Not applicable.

\section{Authors' contributions}

CHL contributed to clinical data analysis and draft of the manuscript. YTL and $\mathrm{CJH}$ had contributions to clinical data acquisition and analysis. MHT had substantial contributions to the conception and design of the study, data analysis, critical revision, and final approval of the manuscript.

\section{Funding}

This work was supported in part by research grants CMRPG8H0751 (CHL) and CMRPG8J0041 (MHT) from Kaohsiung Chang Gung Memorial Hospital, Taiwan. The foundation provided financial support for data collecting and had no further role in study design, analysis, and interpretation of data, the writing of the report, or in the decision to submit the paper for publication.

\section{Availability of data and materials}

The datasets generated and analysed during the current study cannot be made openly due to ethical concern but are available from the corresponding author on reasonable request.

\section{Declarations}

\section{Ethics approval and consent to participate}

This study was approved by the Chang Gung Medical Foundation Institutional Review Board (IRB No.: 104-1961C, 104-2308B, 201800433B0D001, and 201901274B0D001). This study was carried out according to the Declaration of Helsinki. Prior to enrollment, all patients or their legal proxies will be given detailed information about the aims, scope, and possible consequences of the trial by a physician. Written informed consent was obtained from all study participants or their legal proxies.

Consent for publication

Not applicable.

\section{Competing interests}

The authors declare that they have no competing interests.

Received: 27 March 2021 Accepted: 6 September 2021

Published online: 23 September 2021

References

1. Glauser T, Ben-Menachem E, Bourgeois B, Cnaan A, Guerreiro C, Kalviainen $R$, et al. Updated ILAE evidence review of antiepileptic drug efficacy and effectiveness as initial monotherapy for epileptic seizures and syndromes. Epilepsia. 2013;54(3):551-63. https://doi.org/10.1111/epi.12074.

2. Kwan P, Brodie MJ. Early identification of refractory epilepsy. N Engl J Med. 2000;342(5):314-9. https://doi.org/10.1056/NEJM200002033420503.

3. Kwan P, Arzimanoglou A, Berg AT, Brodie MJ, Allen Hauser W, Mathern G, et al. Definition of drug resistant epilepsy: consensus proposal by the ad hoc task force of the ILAE commission on therapeutic strategies. Epilepsia. 2010;51(6):1069-77. https://doi.org/10.1111/j.1528-1167.2009.02397.x.

4. Kwan P, Brodie MJ. Potential role of drug transporters in the pathogenesis of medically intractable epilepsy. Epilepsia. 2005;46(2):224-35. https://doi. org/10.1111/j.0013-9580.2005.31904.X.

5. Fang $M, X i Z Q, W u Y$, Wang XF. A new hypothesis of drug refractory epilepsy: neural network hypothesis. Med Hypotheses. 2011;76(6):871-6. https://doi.org/10.1016/j.mehy.2011.02.039.

6. Rogawski MA, Johnson MR. Intrinsic severity as a determinant of antiepileptic drug refractoriness. Epilepsy Curr. 2008;8(5):127-30. https://doi. org/10.1111/j.1535-7511.2008.00272.x. 
7. Remy S, Gabriel S, Urban BW, Dietrich D, Lehmann TN, Elger CE, et al. A novel mechanism underlying drug resistance in chronic epilepsy. Ann Neurol. 2003;53(4):469-79. https://doi.org/10.1002/ana.10473.

8. Depondt C. The potential of pharmacogenetics in the treatment of epilepsy. Eur J Paediatr Neurol. 2006;10(2):57-65. https://doi.org/10.1016/j.ejpn.2 005.11.009.

9. Abou-Khalil BW. Antiepileptic Drugs. Continuum (Minneap Minn). 2016;22(1 Epilepsy):132-56.

10. Meisler MH, Kearney JA. Sodium channel mutations in epilepsy and other neurological disorders. J Clin Invest. 2005;115(8):2010-7. https://doi.org/1 $0.1172 / \mathrm{JCl} 25466$

11. Tate SK, Depondt C, Sisodiya SM, Cavalleri GL, Schorge S, Soranzo N, et al. Genetic predictors of the maximum doses patients receive during clinical use of the anti-epileptic drugs carbamazepine and phenytoin. Proc Natl Acad Sci U S A. 2005;102(15):5507-12. https://doi.org/10.1073/pnas.040734 6102.

12. Abe T, Seo T, Ishitsu T, Nakagawa T, Hori M, Nakagawa K. Association between SCN1A polymorphism and carbamazepine-resistant epilepsy. Br . Clin Pharmacol. 2008;66(2):304-7. https://doi.org/10.1111/j.1365-2125.2008. 03203.x.

13. Angelopoulou C, Veletza S, Heliopoulos I, Vadikolias K, Tripsianis G, Stathi C, et al. Association of SCN1A gene polymorphism with antiepileptic drug responsiveness in the population of Thrace. Greece Arch Med Sci. 2017. 13(1):138-47. https://doi.org/10.5114/aoms.2016.59737.

14. $\mathrm{Ma} \mathrm{CL}$, Wu XY, Zheng J, Wu ZY, Hong Z, Zhong MK. Association of SCN1A SCN2A and ABCC2 gene polymorphisms with the response to antiepileptic drugs in Chinese Han patients with epilepsy. Pharmacogenomics. 2014; 15(10):1323-36. https://doi.org/10.2217/pgs.14.89.

15. Manna I, Gambardella A, Bianchi A, Striano P, Tozzi R, Aguglia U, et al. A functional polymorphism in the SCN1A gene does not influence antiepileptic drug responsiveness in Italian patients with focal epilepsy. Epilepsia. 2011;52(5):e40-4. https://doi.org/10.1111/j.1528-1167.2011.03097.x.

16. Kumari R, Lakhan R, Kumar S, Garg RK, Misra UK, Kalita J, et al. SCN1AIVS591G-->a polymorphism is associated with susceptibility to epilepsy but not with drug responsiveness. Biochimie. 2013;95(6):1350-3. https://doi.org/10.1 016/j.biochi.2013.02.006.

17. Sanchez MB, Herranz JL, Leno C, Arteaga R, Oterino A, Valdizan EM, et al. Genetic factors associated with drug-resistance of epilepsy: relevance of stratification by patient age and aetiology of epilepsy. Seizure. 2010;19(2): 93-101. https://doi.org/10.1016/.seizure.2009.12.004.

18. Yip TS, O'Doherty C, Tan NC, Dibbens LM, Suppiah V. SCN1A variations and response to multiple antiepileptic drugs. Pharmacogenomics J. 2014;14(4): 385-9. https://doi.org/10.1038/tpj.2013.43.

19. Lakhan R, Kumari R, Misra UK, Kalita J, Pradhan S, Mittal B. Differential role of sodium channels SCN1A and SCN2A gene polymorphisms with epilepsy and multiple drug resistance in the north Indian population. Br J Clin Pharmacol. 2009:68(2):214-20. https://doi.org/10.1111/j.1365-2125.2009.03437.x.

20. Kwan P, Poon WS, Ng HK, Kang DE, Wong V, Ng PW, et al. Multidrug resistance in epilepsy and polymorphisms in the voltage-gated sodium channel genes SCN1A, SCN2A, and SCN3A: correlation among phenotype, genotype, and mRNA expression. Pharmacogenet Genomics. 2008;18(11): 989-98. https://doi.org/10.1097/FPC.0b013e3283117d67.

21. Haerian BS, Baum L, Kwan P, Tan HJ, Raymond AA, Mohamed Z. SCN1A, SCN2A and SCN3A gene polymorphisms and responsiveness to antiepileptic drugs: a multicenter cohort study and meta-analysis. Pharmacogenomics. 2013;14(10):1153-66. https://doi.org/10.2217/pgs.13.104.

22. Brodie MJ. Sodium Channel blockers in the treatment of epilepsy. CNS Drugs. 2017;31(7):527-34. https://doi.org/10.1007/s40263-017-0441-0

23. Abdelsayed M, Sokolov S. Voltage-gated sodium channels: pharmaceutical targets via anticonvulsants to treat epileptic syndromes. Channels (Austin) 2013;7(3):146-52. https://doi.org/10.4161/chan.24380.

24. Claes LR, Deprez L, Suls A, Baets J, Smets K, Van Dyck T, et al. The SCN1A variant database: a novel research and diagnostic tool. Hum Mutat. 2009; 30(10):E904-20. https://doi.org/10.1002/humu.21083.

25. Wolking S, Moreau C, Nies AT, Schaeffeler E, McCormack M, Auce P, et al. Testing association of rare genetic variants with resistance to three common antiseizure medications. Epilepsia. 2020;61(4):657-66.

26. Scheffer IE, Berkovic S, Capovilla G, Connolly MB, French J, Guilhoto L, et al. ILAE classification of the epilepsies: position paper of the ILAE Commission for Classification and Terminology. Epilepsia. 2017;58(4):512-21. https://doi. org/10.1111/epi.13709.
27. Fisher RS, Cross JH, French JA, Higurashi N, Hirsch E, Jansen FE, et al. Operational classification of seizure types by the international leaque against epilepsy: position paper of the ILAE Commission for Classification and Terminology. Epilepsia. 2017;58(4):522-30. https://doi.org/10.1111/epi.13670.

28. Makita N, Sloan-Brown K, Weghuis DO, Ropers HH, George AL Jr. Genomic organization and chromosomal assignment of the human voltage-gated $\mathrm{Na}+$ channel beta 1 subunit gene (SCN1B). Genomics. 1994;23(3):628-34. https://doi.org/10.1006/geno.1994.1551.

29. Catterall WA. From ionic currents to molecular mechanisms: the structure and function of voltage-gated sodium channels. Neuron. 2000;26(1):13-25 https://doi.org/10.1016/S0896-6273(00)81133-2.

30. O'Malley HA, Isom LL. Sodium channel beta subunits: emerging targets in channelopathies. Annu Rev Physiol. 2015;77(1):481-504. https://doi.org/1 0.1146/annurev-physiol-021014-071846.

31. Steel D, Symonds JD, Zuberi SM, Brunklaus A. Dravet syndrome and its mimics: beyond SCN1A. Epilepsia. 2017;58(11):1807-16. https://doi.org/1 0.1111/epi.13889.

32. Baroni D, Moran O. On the multiple roles of the voltage gated sodium channel beta1 subunit in genetic diseases. Front Pharmacol. 2015;6:108. https://doi.org/10.3389/fphar.2015.00108.

33. Uebachs M, Albus C, Opitz T, Isom L, Niespodziany I, Wolff C, et al. Loss of beta1 accessory $\mathrm{Na}+$ channel subunits causes failure of carbamazepine, but not of lacosamide, in blocking high-frequency firing via differential effects on persistent Na+ currents. Epilepsia. 2012;53(11):1959-67. https://doi.org/1 0.1111/j.1528-1167.2012.03675.x.

34. Uebachs M, Opitz T, Royeck M, Dickhof G, Horstmann MT, Isom LL, et al. Efficacy loss of the anticonvulsant carbamazepine in mice lacking sodium channel beta subunits via paradoxical effects on persistent sodium currents. J Neurosci. 2010;30(25):8489-501. https://doi.org/10.1523/JNEUROSCI.1534-1 0.2010.

35. Lucas PT, Meadows LS, Nicholls J, Ragsdale DS. An epilepsy mutation in the beta1 subunit of the voltage-gated sodium channel results in reduced channel sensitivity to phenytoin. Epilepsy Res. 2005;64(3):77-84. https://doi. org/10.1016/j.eplepsyres.2005.03.003.

36. Lek M, Karczewski K, Minikel EV, Samocha KE, Banks E, Fennell T, et al. Analysis of protein-coding genetic variation in 60,706 humans. Nature. 2016 536(7616):285-91. https://doi.org/10.1038/nature19057.

37. Refsgaard L, Olesen MS, Moller DV, Christiansen M, Haunso S, Svendsen JH, et al. Mutation analysis of the candidate genes SCN1B-4B, FHL1, and LMNA in patients with arrhythmogenic right ventricular cardiomyopathy. Appl Transl Genom. 2012;1:44-6. https://doi.org/10.1016/j.atg.2012.06.001.

38. Ricci MT, Menegon S, Vatrano S, Mandrile G, Cerrato N, Carvalho P, et al. SCN1B gene variants in Brugada syndrome: a study of 145 SCN5A-negative patients. Sci Rep. 2014;4(1):6470. https://doi.org/10.1038/srep06470.

39. El-Battrawy I, Muller J, Zhao Z, Cyganek L, Zhong R, Zhang F, et al. Studying Brugada syndrome with an SCN1B variants in human-induced pluripotent stem cell-derived cardiomyocytes. Front Cell Dev Biol. 2019;7:261. https:// doi.org/10.3389/fcell.2019.00261.

40. Schiller Y. Seizure relapse and development of drug resistance following long-term seizure remission. Arch Neurol. 2009;66(10):1233-9. https://doi. org/10.1001/archneurol.2009.211.

41. Callaghan B, Schlesinger M, Rodemer W, Pollard J, Hesdorffer D, Allen Hauser W, et al. Remission and relapse in a drug-resistant epilepsy population followed prospectively. Epilepsia. 2011;52(3):619-26. https://doi. org/10.1111/j.1528-1167.2010.02929.x.

42. Chi X, Li R, Hao X, Chen J, Xiong W, Xu H, et al. Response to treatment schedules after the first antiepileptic drug failed. Epilepsia. 2018;59(11):211824. https://doi.org/10.1111/epi.14565.

43. Spencer CC, Su Z, Donnelly P, Marchini J. Designing genome-wide association studies: sample size, power, imputation, and the choice of genotyping chip. PLoS Genet. 2009;5(5):e1000477. https://doi.org/10.1371/ journal.pgen.1000477.

44. Hirschhorn JN, Lohmueller K, Byrne E, Hirschhorn K. A comprehensive review of genetic association studies. Genet Med. 2002;4(2):45-61. https:// doi.org/10.1097/00125817-200203000-00002.

45. Pitkanen A, Henshall DC, Cross JH, Guerrini R, Jozwiak S, Kokaia M, et al. Advancing research toward faster diagnosis, better treatment, and end of stigma in epilepsy. Epilepsia. 2019;60(7):1281-92. https://doi.org/10.1111/ epi.16091.

46. Balding DJ. A tutorial on statistical methods for population association studies. Nat Rev Genet. 2006;7(10):781-91. https://doi.org/10.1038/nrg1916. 
47. Hendricks AE, Dupuis J, Logue MW, Myers RH, Lunetta KL. Correction for multiple testing in a gene region. Eur J Hum Genet. 2014;22(3):414-8. https://doi.org/10.1038/ejhg.2013.144.

48. Visscher PM, Wray NR, Zhang Q, Sklar P, McCarthy Ml, Brown MA, et al. 10 years of GWAS discovery: biology, function, and translation. Am J Hum Genet. 2017;101(1):5-22. https://doi.org/10.1016/j.ajhg.2017.06.005.

49. Qosa H, Miller DS, Pasinelli P, Trotti D. Regulation of ABC efflux transporters at blood-brain barrier in health and neurological disorders. Brain Res. 2015; 1628(Pt B):298-316.

50. Meng H, Guo G, Ren J, Zhou H, Ge Y, Guo Y. Effects of ABCB1 polymorphisms on plasma carbamazepine concentrations and pharmacoresistance in Chinese patients with epilepsy. Epilepsy Behav. 2011; 21(1):27-30. https://doi.org/10.1016/j.yebeh.2011.02.015.

51. Seo T, Ishitsu T, Ueda N, Nakada N, Yurube K, Ueda K, et al. ABCB1 polymorphisms influence the response to antiepileptic drugs in Japanese epilepsy patients. Pharmacogenomics. 2006;7(4):551-61. https://doi.org/1 $0.2217 / 14622416.7 .4 .551$

52. Ozgon GO, Bebek N, Gul G, Cine N. Association of MDR1 (C3435T) polymorphism and resistance to carbamazepine in epileptic patients from Turkey. Eur Neurol. 2008;59(1-2):67-70. https://doi.org/10.1159/000109264.

53. Ishikawa T, Tsuji A, Inui K, Sai $Y$, Anzai N, Wada M, et al. The genetic polymorphism of drug transporters: functional analysis approaches. Pharmacogenomics. 2004;5(1):67-99. https://doi.org/10.1517/phgs.5.1.67.2 5683.

54. Meyer UA, Zanger UM. Molecular mechanisms of genetic polymorphisms of drug metabolism. Annu Rev Pharmacol Toxicol. 1997;37(1):269-96. https:// doi.org/10.1146/annurev.pharmtox.37.1.269.

55. Hung CC, Chang WL, Ho JL, Tai JJ, Hsieh TJ, Huang HC, et al. Association of polymorphisms in EPHX1, UGT2B7, ABCB1, ABCC2, SCN1A and SCN2A genes with carbamazepine therapy optimization. Pharmacogenomics. 2012;13(2): 159-69. https://doi.org/10.2217/pgs.11.141.

56. $M a C L$, Wu XY, Jiao Z, Hong Z, Wu ZY, Zhong MK. SCN1A, ABCC2 and UGT2B7 gene polymorphisms in association with individualized oxcarbazepine therapy. Pharmacogenomics. 2015;16(4):347-60. https://doi. org/10.2217/pgs.14.186.

57. Orsini A, Zara F, Striano P. Recent advances in epilepsy genetics. Neurosci Lett. 2018:667:4-9. https://doi.org/10.1016/.jneulet.2017.05.014.

58. Myers $\mathrm{CT}$, Mefford HC. Advancing epilepsy genetics in the genomic era. Genome Med. 2015;7(1):91. https://doi.org/10.1186/s13073-015-0214-7.

59. Haerian BS, Baum L, Tan HJ, Kwan P, Raymond AA, Saruwatari J, et al. SCN1A IVS5N+5 polymorphism and response to sodium valproate: a multicenter study. Pharmacogenomics. 2012;13(13):1477-85. https://doi. org/10.2217/pgs.12.127.

60. Jang SY, Kim MK, Lee KR, Park MS, Kim BC, Cho KH, et al. Gene-to-gene interaction between sodium channel-related genes in determining the risk of antiepileptic drug resistance. J Korean Med Sci. 2009;24(1):62-8. https:// doi.org/10.3346/jkms.2009.24.1.62

61. Torkamani A, Wineinger NE, Topol EJ. The personal and clinical utility of polygenic risk scores. Nat Rev Genet. 2018;19(9):581-90. https://doi.org/10.1 038/s41576-018-0018-x.

62. Mega JL, Stitziel NO, Smith JG, Chasman DI, Caulfield M, Devlin JJ, et al. Genetic risk, coronary heart disease events, and the clinical benefit of statin therapy: an analysis of primary and secondary prevention trials. Lancet. 2015;385(9984):2264-71. https://doi.org/10.1016/S0140-6736(14)61730-X.

\section{Publisher's Note}

Springer Nature remains neutral with regard to jurisdictional claims in published maps and institutional affiliations.

Ready to submit your research? Choose BMC and benefit from:

- fast, convenient online submission

- thorough peer review by experienced researchers in your field

- rapid publication on acceptance

- support for research data, including large and complex data types

- gold Open Access which fosters wider collaboration and increased citations

- maximum visibility for your research: over $100 \mathrm{M}$ website views per year

At $\mathrm{BMC}$, research is always in progress.

Learn more biomedcentral.com/submissions 